\title{
Influential Factors on Human Performance in Railways and their Interrelations
}

\author{
T. Lindner \& B. Milius \\ Institute of Railway Systems Engineering and Traffic Safety \\ Technische Universität Braunschweig, Germany \\ D. Schwencke \& K. Lemmer \\ Institute of Transportation Systems, German Aerospace Center, Braunschweig, Germany
}

\begin{abstract}
In this paper performance shaping factors (PSFs) that occurred most frequently in the analysis of signals passed at danger (SPADs) with the second generation method CAHR are discussed. In order to assess the validity of the identified PSFs, we compare our results with the results obtained from an adapted Why-Because-Analysis of a subset of the same incidents. The relationship and dependencies between the factors are discussed in detail. We put the identified PSFs for SPADs in the context of an existing PSF taxonomy, in the context of a simple model of the railway system and perform a clustering on them. In the last part of the paper we explain how future simulation studies can help to advance in the understanding of the identified PSFs and their relationships.
\end{abstract}

\section{INTRODUCTION}

In human reliability analysis it is always of major concern to identify and quantify performance shaping factors (PSFs) correctly, since those may affect results of reliability assessments considerably. There are many different approaches to use PSFs in existing assessment methods. Note that in some of them, alternative names are used for the concept of PSFs, for example "performance influencing factors" (SHERPA method) or "error producing conditions" (HEART method). One reason for the variety in the use of PSFs is the fact that they often lack a sufficiently detailed definition and a clear scope. Multiple interdependencies between PSFs furthermore lead to difficulties in distinguishing between them and make PSFs hard to handle. This leaves room for misunderstanding and interpretation.

In Boring et al. (2007) performance shaping factors are defined as " ...influences that enhance or degrade human performance and provide basis for considering potential influences on human performance and systematically considering them in quantification of human error probabilities (HEPs)". They are further categorized as internal and external with "internal PSFs - influences that the individual brings to the situation such as mood, fitness, stress level, etc." and "external PSFs - influences in the situation or environment that affect the individual such as temperature, noise, work practices, etc." It is also possible to classify PSFs in direct and indirect PSFs. Di- rect PSFs are defined as that they "can be measured directly, whereby there is a one-to-one relationship between the magnitude of the PSF and that which is measured". Indirect PSFs are described as that they "cannot be measured directly, whereby the magnitude of the PSF can only be determined multivariately or subjectively".

Even though the definitions do serve as a first guideline, their practical application in order to obtain a clear structure in the end is rather difficult. Furthermore it is necessary to understand exactly how PSFs influence human actions to get a vivid impression of this relationship. Only then PSFs can be easily applied in the railway context. It is desirable to define a hierarchical structure for PSFs to keep the interdependencies at a minimum as otherwise an influence might get too much weight.

In this paper, we will first give an overview about what PSFs are. We will discuss the aspects which make dealing with PSFs difficult. The experience gathered in the DFG-funded project SMSmod will be used to paint a clear picture of PSFs in railway incidents. In particular we identify a set of most frequent PSFs that have an influence on the train driver. In this paper we will focus on the incident "signal passed at danger" (SPAD) as this incident was by far the one found most often in the database. Additionally, as this hazard is very well researched e.g. in the UK, SPADs allow to compare the results with other work (e.g. Kyriakidis et al., 2012).

The PSFs found in the incidents analyzed in SMSmod were identified using two different ap- 
proaches: One approach is the second generation human reliability assessment method CAHR, the other one is the accident analysis method "WhyBecause-Analysis" (WBA). These two methods were applied to incidents from the data base provided by the German railways. The most important PSFs, identified as results are presented in the following sections. Conclusions towards defining and structuring PSFs in a systematic way are drawn. Finally, the possibility of a detailed assessment of the most influential PSFs in the context of the train driver is discussed.

\section{PERFORMANCE SHAPING FACTORS}

As described in the introduction, PSFs influence human behavior and human reliability. If PSFs are not taken into account when human reliability is assessed, the results might significantly differ from reality.

Generally, PSFs are most often thought of as a negative influence on human behavior. This means, that they usually describe an influence which decreases the operator's reliability. Nevertheless, depending on how a PSF is "set on the scale", it may also have a beneficial effect on human reliability. Furthermore in general there is no simple relationship between the presence of the PSF and the increase or decrease of human reliability, e.g. for the PSF stress it is known that too much stress but also mental underload lead to a decrease in reliability.

The difficulties arising from identifying PSFs and their inclusion in a human factors analysis are summarized in the following section.

\section{Incident descriptions influencing results}

Depending on the degree of detail of a description of a given incident it can be very difficult to derive specific performance shaping factors. Usually, an incident description does not contain specific information about the influencing factors leading to a hazardous event, but only a description of the actions / mistakes of the persons analyzed as well as obvious (technical) information. Our experience from the project SMSmod has shown that only in some cases PSFs could be taken directly from incident description. Usually, such PSFs are irregularities in operations, daytime/nighttime or driver experience. Many other aspects leading to an incident can only be assumed as they are not literally discussed in the description. The reason for this might be the shortcomings of guidelines for incident descriptions as these set their focus on the results of the incidents, rather than on the underlying causes. Another reason might be that especially PSFs which are directly related to the human cannot be assessed easily because e.g. the human is not aware of them and will therefore not mention them in incident descriptions. To summa- rize the above, deriving PSFs from incident descriptions one may miss important PSFs which are not addressed directly in the descriptions. Methods are needed which help to identify PSFs in a systematic manner.

\section{Definition and overlap of different PSFs}

Different persons might define PSFs differently. Currently no generally accepted and well defined PSF taxonomy appears to exist. Furthermore, PSFs are often closely related which makes it difficult to distinguish one from another. A very good example for this is a PSF "irregularity in operation". This PSF does probably also relate to PSFs like distraction, knowledge of rules or stress. Another example is the difficulty of distinguishing the general term "stress" from the more specific "workload". In both examples a user of a given PSF taxonomy can choose any of the given terms and be correct, no matter which one he chooses. Such inconsistencies make it difficult to produce reliable results. Therefore, strict definitions which are generally applicable in railways are needed. This criticism is closely connected to the task of setting strict boundaries between different PSFs. Furthermore, it is necessary to set up a hierarchical structure which displays the dependencies of the PSFs on e.g. different levels of detail.

\section{Effects of PSFs}

The effect that a PSF has on a person cannot easily be calculated as it might vary widely depending on e.g. personal experiences and interdependencies with other PSFs occurring. Therefore for e.g. risk assessments it is difficult to capture and quantify the effect a PSF has on a human as part of a system.

To get a better understanding of the impact of certain PSFs, it is crucial to analyze as much data as possible to get qualitative and especially quantitative information about the impact a PSF can have. A concept is needed to deal with a large variety of possible effects of PSFs on humans in analyses.

\section{ANALYZING PERFORMANCE SHAPING FACTORS WITH CAHR}

CAHR is the acronym for "Connectionism Assessment of Human Reliability". With the database system CAHR (Sträter 2000) it is possible to analyze operational disturbances caused by human actions. It is based on a generic knowledge base for the incident analysis that is extendable by the description of further incidents. The knowledge-base e.g. contains information about the system state.

In contrast to past methods for incident analysis, the CAHR evaluation process is an analytical and not a classifying method. In the method, an incident is systematically broken down, analyzed, and described for the purpose of a qualitative error and cause de- 
termination. The analysis method proceeds from general questions to substantive information that was observable during the incident via actions and it moves on to error indications and PSFs. Although the method employs existing taxonomies from literature for support during analysis, it is not tied to any fixed, predetermined descriptors. It is, in other words, an open procedure. The approach makes it possible to preserve the original information of the incidents. Besides, there is no compulsion to categorize an incident in a certain classification scheme that might possibly not reproduce actually observed aspects correctly and that could thus lead to misinterpretations.

The described general procedure of CAHR is also applicable for the determination of PSFs in CAHR.

PSFs are derived from hazardous incident descriptions and have to be manually chosen for each incident. There is no fixed taxonomy that has to be applied in CAHR.

Within the work on the SMSmod project a large number of PSFs were identified. As some PSFs were only identified once in the course of 105 descriptions of SPADs, it does not seem sensible to take into account all PSFs that influence the train driver. We will therefore focus only on the PSFs that were identified most often in SPADs. These PSFs are given in Table 1, where all PSFs except comprehension have negative influence on the train driver's reliability. (Note that the quantities do not add up to 105 because one incident may be influenced by more than one PSF.)

Table 1. Most frequent PSFs identified in connection with SPADs using CAHR

\begin{tabular}{lc}
\hline Performance Shaping Factor & Quantity \\
\hline irregularities & 52 \\
distraction & 50 \\
comprehension (positive) & 40 \\
visibility conditions & 36 \\
fatigue & 27 \\
job experience & 27 \\
just culture & 13 \\
signal location & 10 \\
stress / strain & 9 \\
signal design & 7 \\
\hline
\end{tabular}

\section{ANALYZING PERFORMANCE SHAPING FACTORS WITH WHY BECAUSE ANALYSES}

Why-Because Analysis (WBA) is a well established causal incident analysis method (Ladkin 2001). A top event is broken down step by step to a complete set of necessary causes for the top event; this way a why-because-graph is created. In order to ensure these properties of the causes, in each step counterfactual tests and a causal completeness test have to be carried out. Being a rather formal method, WBA guides the applicant to exhibit all causes of an incident, regardless whether they are attributed to an individual, an organization, a technical system or the environment. It avoids including speculative causes, meaning that each cause identified actually influenced the outcome (the top event). It encourages to discuss the relations of causes in detail, to logically derive causes and to search for missing pieces of information.

On the other hand, this also means that the application of the method is rather conservative, as it is very dependent on the availability of facts about the incident, i.e. the analysis may be very limited if not all of the main contributing causes are known. Especially human actions (or omitted actions) can often not be further analyzed, since it is not always clear what lead the human to a particular decision. For the latter reason we attached PSFs to the causes identified in WBAs, where we used a structured list of PSFs (Schwencke, Talg \& Lemmer 2012) created mainly through a literature survey within the SMSmod project as a basis. The PSFs could either directly be encoded in the cause (e.g. if the WBA already found out that the weather conditions lead to a prolonged breaking distance which in turn was a cause for a SPAD) or refer to a fact associated with the cause which was known but could not be included in the WBA because of the formal restrictions (e.g. it may be known that it was dark outside when the train driver overlooked a sign, but this is not a complete set of causes). It can then be read off from the whybecause-graph which PSFs (assigned to root causes) influenced which human action (given as a cause in the graph).

We investigated eight SPAD incidents for which there was sufficient information to conduct a WBA and assigned PSFs as described above. Table 2 shows the performance shaping factors that were found most often. For the interpretation of the counts please note that

- the PSFs of the whole incident were counted (in two cases the SPADs were followed by further errors);

- a PSF was counted several times for one event in case it influenced several causes;

- only PSFs that influenced the train driver were taken into account; and

- it was distinguished between negative and positive influences (displayed in the two columns). 
Table 2. Frequent performance shaping factors in connection with SPADs identified using WBAs

\begin{tabular}{lcc}
\hline Performance Shaping Factor & $\begin{array}{c}\text { Quantity } \\
\text { (neg) }\end{array}$ & $\begin{array}{c}\text { Quantity } \\
\text { (pos) }\end{array}$ \\
\hline night hour & 7 & 0 \\
darkness & 6 & 0 \\
irregularities & 5 & 1 \\
communication & 5 & 0 \\
trackside complexity & 5 & 0 \\
attention & 4 & 2 \\
distraction (by persons) & 4 & 0 \\
feedback from technical system & 3 & 2 \\
bad sight (weather / sun) & 3 & 0 \\
signal design & 2 & 0 \\
\hline
\end{tabular}

\section{COMPARISON OF PERFORMANCE SHAPING FACTORS IDENTIFIED IN CAHR AND WITH WBA}

Comparing the approaches to determining the most important PSFs via CAHR and WBAs, it should first be stated that both methods were conducted using incidents from the same database. However, the number of incidents that were investigated significantly differed between the two methods (eight WBAs for SPAD incidents and 105 SPAD incidents in CAHR which included the former). In contrast to the CAHR evaluation, for the assignment of PSFs in the WBAs a previously fixed taxonomy (Schwencke, Talg \& Lemmer 2012) based on a literature study was used. Thus different sets of PSFs were assigned using two different incident analysis methods in order to explore a wide range of PSFs and to obtain solid insights how they affect railway operation.

In general, the WBA results from Table 2 confirm the CAHR-findings from Table 1: irregularities are a major influence in both cases as well as distraction, where the lower position in Table 2 reflects the fact that the entry is restricted to distraction by persons. The PSF "fatigue" was chosen in the CAHR approach whenever the incident took place at night, so this corresponds to the WBA-PSF "night hour". The "visibility conditions" from Table 1 comprise the entries "darkness" and "bad sight (weather / sun)" from Table 2. A smaller but still significant impact of the signal design is stated in both tables.

There are also some differences in the PSFs obtained from the two approaches: the CAHR-PSFs "comprehension (positive)", "job experience", "just culture", "signal location" and "stress" do not occur in Table 2. Comprehension is the only positive PSF which was found to be significant for SPADs in CAHR; since the comprehension of the situation by the train driver may be related to different or even no perceptions, it may well be that it matches all of the posi- tive occurrences of PSFs found in Table 2. A more detailed comparison of the positive CAHR- and WBA-PSFs cannot be given due to the different approaches (single positive PSFs in CAHR vs. potential positive influences of all WBA-PSFs). In the case of job experience, the difference between CAHR and WBA is due to a more fine-grained approach in case of the WBAs, where several related PSFs concerning the familiarity with different aspects of train and infrastructure were used. Just culture was not part of the PSF taxonomy used for the WBA analyses since there can hardly be found evidence in the literature that this is an issue in railway incidents; it is however discussed as a factor related to reporting safety issues in the UK. Nevertheless, the incident reports suggest that it might have led train drivers to act in a way so that they would not be blamed for their mistakes, so it was included in the CAHR-PSFs. In the case of signal location the difference most likely is due to a different interpretation of the incident data from which an influence by the signal location can seldom be read off directly, but seems to be likely in some cases. This may also have contributed to the considerable score of the "trackside complexity" WBA-PSF although there was a separate WBA-PSF which directly refers to the signal position. Stress, as it may originate from many different sources, was not chosen as a PSF itself in the WBA approach but can be inferred from other PSFs.

There are also several WBA-PSFs (communication, trackside complexity, attention and feedback from technical system) which do not occur in the list of the most important CAHR-PSFs. In case of "communication" and "feedback from technical system" these PSFs only occurred in a small number of cases so that they do not appear in Table 1. The trackside complexity score was partly explained above. Attention as a very dependent measure was not included in the CAHR PSFs.

In conclusion, both approaches complement each other. Some major PSFs are confirmed by both approaches, others are found to be very influential only by one method which is either due to a different abstraction level of PSFs (one PSF in one method corresponding to many PSFs in the other method) or a different interpretation or assignment of the PSFs. Despite the relative small sample the WBAs added further insights to the CAHR results, e.g. emphasized the importance of communication in train driving. Working with a comprehensive list of PSFs as in the WBA approach which prevents leaving PSFs aside which cannot be read off from the incident reports at first sight seems to be of advantage only for identifying less important PSFs and when a larger sample is inspected. As the reader may have noticed from the comparison of the different sets of PSFs, it 
is crucial to have precise definitions of the PSFs to be able to relate them properly. One step towards getting a clear picture of what a PSF means is discussed in the following chapter, where the PSFs discussed above are classified and grouped under different aspects.

\section{CLASSIFICATION OF PERFORMANCE SHAPING FACTORS}

PSF taxonomies are given - besides the ones in HRA methods - e.g. by Kyriakidis (2012), in the German standard VDI 4006 (1999) and by Hammerl (2011). The railway specific classification described by Hammerl distinguishes physical, personal and organizational PSFs. His focus was on minimizing mutual influences of his set of PSFs, i.e. to provide PSFs which are as independent as possible. Since dependencies seemed to be inevitable in case of the influence of some organizational factors (e.g. training) on some personal factors (e.g. expertise), he further split up personal factors into individual factors and influenced factors and organizational factors into basic and staff-related factors. Clustering our PSFs from Tables 1 and 2 according to his framework results in Table 3 .

Table 3. Clustering the identified PSFs with Hammerl's classification

\begin{tabular}{cl} 
& visibility conditions \\
& darkness \\
bad sight \\
trackside complexity \\
feedback from technical system \\
& signal design, signal location \\
\hline Personal & \\
individual & \\
\hline Personal & fatigue / night hour \\
influenced & job experience \\
\hline Organizational & \\
staff-related & \\
\hline Organizational & \\
basic & \\
\hline
\end{tabular}

The personal factors communication, attention and stress do not match the influences listed by Hammerl (2011) under his categories, since they are dependent on many different factors. Irregularities and distraction do not fit well in his framework. Moreover, both of them may have many very different origins.

The clustering in Table 3 suggest that SPADs are mainly influenced by factors from two groups. The large number of physical factors indicates that a more user-friendly design and alignment of technical elements of the railway system could improve the situation w.r.t. SPADs - even sight-related factors may be dealt with by changes in technical systems as replacing trackside elements by in-cabin displays. The personal factors which fit and those which do not fit in the classification of Table 3 emphasize that the relevant personal factors are influenceable and are dependent on many different aspects. Thus in order to reduce the negative influence of those factors on SPADs a comparatively expensive holistic approach including the consideration of many further underlying personal and organizational factors needs to be taken.

The fact that no organizational factors were found among the most important PSFs reflects that organizational matters influence railway operation in an indirect way, but also that there seems to be no closer look at and documentation of organizational causes during incident investigations.

Especially irregularities, distraction and stress, which were identified as some of the most frequent influencing factors by CAHR as well as by WBA analyses do not fit with the classification of Hammerl. This leads to the question, if it might be possible to classify the PSFs on the basis of their sources and dependencies. In the following sections we discuss two different approaches which may be of interest in order to get a deeper understanding of what are relevant issues for human reliability in railways and where these issues are located in the railway system. Firstly, based on the organizational structure of railway operation, a classification of PSFs according to the different parts of the railway system aiming at a straight forward application is given. Secondly, a clustering purely based on the CAHR and WBA evaluation results is performed.

\section{Classification of Performance Shaping Factors from the structure of railway operation}

The results of the evaluation of incident reports using CAHR and WBA has shown that very many different PSFs can be identified. It also shows that the question which PSFs are the "correct" ones remains and also if all relevant PSFs are identified. Considering the high number of PSFs that can be found it is rather difficult to stay focused on the ones which might influence the safety of the railway system most. Therefore, our approach focusses on PSFs which have a direct influence on the most basic function of the railway system that is providing a safe train ride.

The railway system itself is complex, as a safe train ride is only possible when very different parts of the railway system and the environment are working together. Most basically, the railway system can be divided into the different subsystems of railway infrastructure and operation (e.g. signals, infrastructure elements and their operators) and the train driver. The environment comprises e.g. landscape and 
weather. The simplified classification system view is shown in Figure 1.

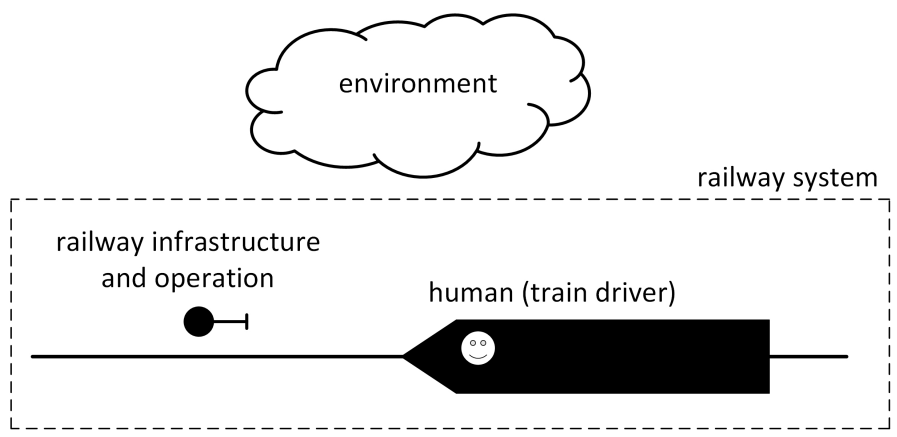

Figure 1. Different system parts working together

A basic rule for allocating the following PSFs is that a PSF can only be assigned to the subsystem in which it can be directly influenced. Furthermore, the PSF is to have a direct influence on the train ride as such. These PSFs are called primary PSFs. One example of a primary PSF is signal location. A signal location which makes it difficult to see the signal can be changed directly at the signal. Missing a signal or recognizing it too late has direct impact on the train ride. For most PSFs given in Table 1 such an allocation was carried out and is shown in Figure 2.

The PSFs in the middle of Figure 2 (distraction and stress / strain) cannot be allocated to any subsystem. Such influencing factors are called secondary PSFs. Rather than originating in a subsystem they describe the effect (primary) PSFs can have on the human.

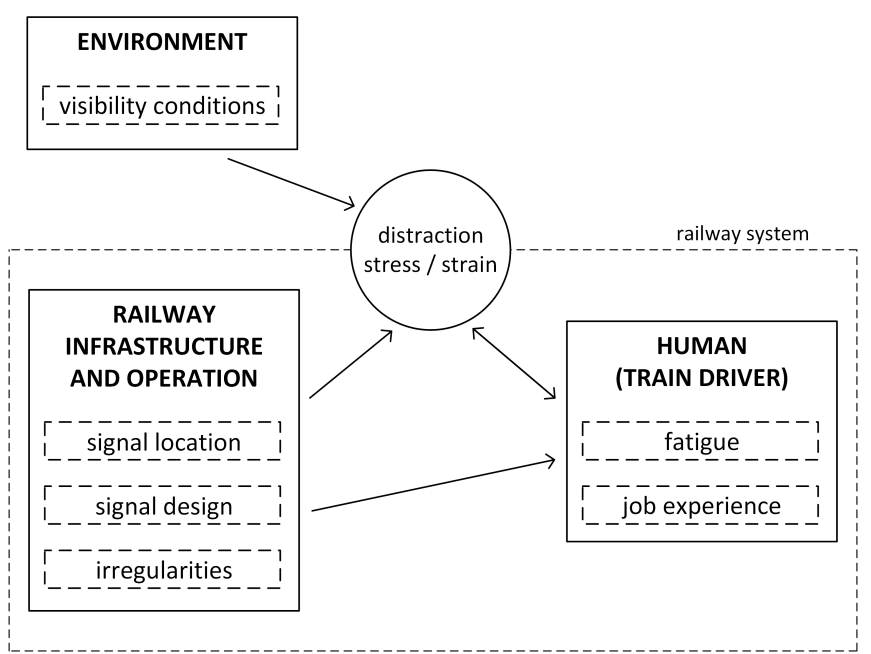

Figure 2. Classification of PSFs to the different subsystems

Both PSFs - distraction and stress / strain - describe an effect of the primary PSFs on the human e.g. of seeing a signal aspect too late. Also, primary human PSFs can affect the human behavior via secondary
PSFs. As an example, lack of job experience might lead to increased stress.

Not all PSFs which were identified using CAHR or WBA classify as PSFs in our proposed structure. An example is age which is sometimes labeled as PSF as well. In our classification, age would be considered as a cause to several PSF e.g. fatigue or (bad) sight. Another example for aspects not included are most aspects related to the organization such as e.g. just culture or hierarchical order as these do not have a direct impact on safety. In the focus of the classification is the identification of means which enable safe train rides. As such, only aspects which impact a train ride directly are called PSFs. When an analysis shows that some aspects influence safety more than others, a detailed analysis of these PSFs will show the very different reasons for the bad impact of the factor. Such an analysis which will look at very different sources of information and give the user an idea how to influence the problematic PSF to reach a higher safety level. By following the proposed two step approach it allows the user to focus on aspects which are especially relevant in the given context.

As a first conclusion of this we can postulate that by including secondary PSFs together with primary PSFs by which they are influenced in experiments or in the evaluation of incidents we will result in an overlay of negative influences on human behavior as the same effect, e.g. an irregularity (a primary PSF), is weighted stronger since it is also included in the (secondary) PSF stress. A division into primary and secondary PSFs may be a good possibility for handling the problem of the dependencies between different factors. We will need to look at more examples to find out of the given structure of the railway system needs to be expanded. Also, we have to transfer all PSFs identified into our classification to get more experience with its application.

\section{Clustering of the Performance Shaping Factors from the CAHR and WBA results}

Apart from general scientific PSF taxonomies, it seemed scientifically interesting to provide a free topical clustering of the most important PSFs related to SPADs that we found, in order to obtain a highlevel view on the situation. We produced such a clustering without using a particular method like factor analysis or cluster analysis (Bortz 2005), but just by sorting the PSFs from Tables 1 and 2 into the structure of the PSF-list which was used for assigning PSFs to WBAs. With this as a basic clustering, in a second step it was tried to form as few groups of PSFs as possible, where the PSFs in one group are still arguably related. This led us to consider the four critical groups of PSFs shown in Table 4. 
Table 4. Clustering the most important PSFs into groups

\begin{tabular}{|c|c|c|c|}
\hline No. & Group & Factors & Comment \\
\hline 1 & $\begin{array}{l}\text { personal } \\
\text { factors }\end{array}$ & $\begin{array}{l}\text { fatigue / night hour, } \\
\text { communication, attention, } \\
\text { job experience }\end{array}$ & $\begin{array}{l}\text { A wide range of activities can be thought of to improve the situation, from very } \\
\text { basic (shape positive culture) to very specific (provide more operational and } \\
\text { environmental information to the train driver). The underlying organizational } \\
\text { and related personal factors must be considered and actions taken may affect any } \\
\text { role, procedure or technical system. }\end{array}$ \\
\hline 2 & $\begin{array}{l}\text { unusual } \\
\text { events }\end{array}$ & $\begin{array}{l}\text { irregularities, distraction, } \\
\text { stress }\end{array}$ & $\begin{array}{l}\text { It seems that it is difficult for the train driver to handle disturbances. The } \\
\text { reasons for this may be manifold (out of the loop-phenomenon due to } \\
\text { automation, normally operation based on strict rules, missing information about } \\
\text { context of own train ride). This highlights the importance of resilience } \\
\text { engineering, i.e. to design the railway system more flexible in order to cope with } \\
\text { unexpected events. This includes procedures, systems design and humans as } \\
\text { well. }\end{array}$ \\
\hline 3 & $\begin{array}{l}\text { bad } \\
\text { visibility }\end{array}$ & $\begin{array}{l}\text { visibility conditions / bad } \\
\text { sight (weather / sun) / } \\
\text { darkness }\end{array}$ & $\begin{array}{l}\text { Not much of a surprise for a visual task like perceiving a red signal aspect. } \\
\text { Many technical solutions can be thought of in order to mitigate the influence of } \\
\text { the factors considerably, e.g. in-cabin displays. }\end{array}$ \\
\hline 4 & $\begin{array}{l}\text { systems } \\
\text { design }\end{array}$ & $\begin{array}{l}\text { trackside complexity, } \\
\text { feedback from technical } \\
\text { system, signal design, } \\
\text { signal location }\end{array}$ & $\begin{array}{l}\text { This pertains to infrastructure and in-cabin systems. It exhibits potential for } \\
\text { applying usability criteria, considering human factors and involving the user } \\
\text { when designing railway systems. }\end{array}$ \\
\hline
\end{tabular}

For all of the groups it is also important to raise the train driver's awareness of these factors and to provide possible strategies to cope with them.

In order to check whether the clustering has produced groups of particularly interrelated PSFs, from the CAHR data an analysis of the common occurrences of PSFs can be performed.

\section{SUMMARY AND OUTLOOK}

Assessing human reliability, it is necessary to take PSFs into account. The experience from the project SMSmod has shown that many influencing aspects can only be assumed as they are not discussed in incident descriptions. There may also exist overlaps of different PSFs, so it is hardly possible to assign a factors to an incident without discussion. To minimize these problems, one important step is to give strict definitions of each PSF. The complexity of calculating the effect of PSFs on a person requires not only as much evaluation data as possible, but also clear concepts.

We presented two different approaches (CAHR and WBA) to the identification of the most important performance shaping factors in relation with SPADs from incident data. The results of the WBAs mostly confirmed but also complemented the ones from the CAHR analysis. We found several advantages of the additional use of WBAs for the assignment of PSFs to incidents: the way in which the PSFs influence the incidents is explicit in the whybecause-graph, and a Why-Because Analysis filters the pieces of information about the incident which arguably caused it and helps to find out about missing pieces of such information. Thus the PSFs assigned should be less speculative. Disadvantages lie in the considerable effort to create a WBA and in that the formal restrictions of the method may rule out some incomplete but nevertheless interesting detail information from the why-because-graph and thus also prevent the assignment of the PSFs related to this information.

One PSF that is of particular interest in railway operation is "distraction". Distraction was found to be one root cause in the majority of the SPAD's that occurred in the past. In PSF research, the concept of "distraction" suffers a lack of definition and often covers different kinds of distractors that are qualitatively quite different from each other. Distraction can be external in nature, when something unexpected happens in the environment of the driver, but it can also be more internal, like distracting thoughts that impair attentional processes. Within the external origins of distraction, a further distinction should be made between distraction inside and outside the driver's cab, as well as between distractors that are out of the driver's control and deliberate selfdistraction (like answering a private phone call). Many more distinctions are imaginable, but a more systematical definition of the concept of distraction is mandatory and will lead to detailed research questions.

Distraction as well as stress / strain and irregularities, as other examples of the most frequent factors, are not considered in the classification of Hammerl (2011) which aimed at providing a small set of rather 
independent PSFs. This together with the need for clearly defined and independent PSFs led to the development of a classification of the identified most important SPAD-related PSFs according to the organizational structure of railway operation. In this approach, influencing factors are divided into primary and secondary PSFs. This kind of classification might help to differ between factors that result from properties of the system or human influences as well as factors that are influenced or rather caused by the appearance of other, superior (primary) PSFs. These results are helpful to understand how the most important PSFs act in the context of the railway system. Moreover, the assignment of PSFs to different parts of the railway system may facilitate the application of the results about important PSFs (e.g. for SPADs) by the railway industry. Our free clustering may support this as it provides a view that is more focused on potential spheres of activity. Such high-level view can help to find holistic strategies to improve human reliability in connection with SPADs. The presented approach is easy to understand and allows a user e.g. engineers a more systematic approach to the application of PSFs especially with regard to overlapping effects of different PSFs.

The set of identified major PSFs for the train driver during SPADs should be investigated in future studies. Especially the laboratory environment of a train simulator offers unique possibilities for a variety of research. Safety impairing influences of negative PSF's under safe and controllable conditions can be systematically analyzed under realistic conditions. For example, irregular and uncommon situations in regular railway operation can be introduced to train drivers, in order to comprehend their problem solving strategies and to evaluate the resilience of the human-machine interaction in railway operation.

Finally, simulator studies can help to study in depth presumable dependencies of PSFs. This may be useful e.g. in order to validate CAHR interdependency results and to answers the question about the correlations between primary and secondary PSFs or the groups of PSFs related to the different parts of the railway system. Such investigation may also serve to provide a quantitative extension of the classification approaches. It will also be possible to relate to a variety of psychological concepts that can serve as an explanatory framework for SPADs at a deeper level. Situation awareness and vigilance are promising concepts that could be used to explain the influence that negative PSF's either have or do not have on attention.
The CAHR and WBA evaluations presented in this paper were conducted in the project "SMSmod" funded by the German Research Association (DFG).

\section{REFERENCES}

Boring, R.L., C.D. Griffith, and J.C. Joe (2007) The Measure of Human Error: Direct and Indirect Performance Shaping Factors, in: joint 8th IEEE Conference on Human Factors and Power Plants / 13th Conference on Human Performance, Root Cause and Trending (IEEE HFPP \& HPRCT). 2007, Idaho National Laboratory.

Bortz, J. (2005) Statistik für Human- und Sozialwissenschaftler. 6. Auflage, Springer.

Hammerl, M. (2011) Analyse der menschlichen Einflussfaktoren und Zuverlässigkeit im Eisenbahnverkehr. Phd thesis, Technische Universität Braunschweig.

Kyriakidis, M. et al. (2012) The development and assessment of a performance shaping factors taxonomy for railway operations. In: Journal of the Transportation Research Board, Vol. 2289 (2012), 145-153.

Ladkin, P. B. (2001) Causal System Analysis. RVS-Bk-05-01. Bielefeld: University of Bielefeld, RVS Group.

Schwencke, D., Talg, M. \& Lemmer, K. (2012) Bewertung des Einflusses von Performance Shaping Factors auf menschliche Handlungen im Bahnbetrieb. In Proc. Frühjahrskongess 2012 der Gesellschaft für Arbeitswissenschaften Kassel.

Sträter, O. (2000) Evaluation of Human Reliability on the Basis of Operational Experience. GRS-170. Köln: Gesellschaft für Anlagen- und Reaktorsicherheit.

VDI 4006 (1999) Menschliche Zuverlässigkeit - Teil 1: Ergonomische Forderungen und Methoden der Bewertung - Teil 2: Methoden zur quantitativen Bewertung menschlicher $\mathrm{Zu}$ verlässigkeit. Beuth-Verlag. Berlin. 